\title{
Segregación Urbana y Políticas Públicas con Especial Referencia a América Latina
}

\author{
JAIME AYMERICH*
}

Este artículo explora políticas públicas en el contexto de América Latina en lo relativo a segregación socioespacial, entendiendo que la noción de políticas públicas se refiere a orientaciones generales que un gobierno o un sector del aparato público establecen con respecto a un problema de interés para la sociedad o un grupo de personas.

En segundo lugar las políticas públicas a las que se hace referencia son las políticas urbanas, tanto las que inciden de manera directa o indirecta en la producción de la segregación socioespacial como las políticas que se orientan a revertirla. También se incluyen algunas referencias a la acción del mercado que en un contexto neoliberal asume un rol dominante en dichos procesos.

La noción de segregación socioespacial y algunas de sus determinaciones.

El propósito de entrar en algunas consideraciones conceptuales es que ellas puedan precisar significados de las políticas urbanas que tienen implicancias directas o indirectas con la segregación socioespacial.

Segregar según el diccionario significa separar una cosa de otra o de otras y segregación urbana alude a la forma como se separan los elementos de un todo que es la aglomeración urbana. , ciudad formal versus ciudad informal, ciudad integrada versus ciudad marginal $y /$ o fragmentada.

La separación de los elementos de la totalidad urbana puede asociarse a la lógica de la división funcional del espacio y a las lógicas de la división social del espacio.

La división funcional. del espacio comprende la localización diferenciada de actividades y funciones en el espacio urbano; de producción; de intercambio de per-

* Profesor Departamento de Sociología. Universidad de Chile 
sonas bienes y servicios; de gestión; de consumo del espacio, tanto residencial como de equipamientos colectivos, en tanto que la división social espacial hace referencia solamente a un aspecto de la división funcional , al consumo residencial del espacio como ocupación de aquel por grupos sociales distintos y la segregación significa en este caso la organización del espacio en zonas residenciales de fuerte homogeneidad interna y una fuerte disparidad social entre ellas. . En un sentido amplio hablamos de segregación socioespacial para referirnos a la diferenciación residencial en la totalidad urbana según criterios diversos; división socio-económica del espacio, incluyendo tanto los agrupamientos sociales positiva o negativamente privilegiados, la segregación demográfica y la segregación étnico/racial para señalar los más significativos. Cuando empleamos el término segregación socioespacial en un sentido restringido lo referimos a espacios de fuerte homogeneidad interna cuyos atributos son la pobreza y la exclusión.

Si hacemos esta distinción entre estas dos significaciones de la segregación es porque esta aparece como problemática de dos maneras, por una parte, en relación a la ciudad como totalidad, como mosaico de segmentos sociales espacialmente diferenciados, la ciudad en fragmentos, y por otra, en relación a los grupos sociales negativamente privilegiados espacialmente segregados. Cuando la segregación definida en un sentido amplio se instala como tendencia en el desarrollo urbano entraña la disolución de las relaciones sociales y lo que algunos autores denominan la "crisis " de la ciudad (Léfebvre, 1969) o su"decadencia" (Touraine, 1998.) . En la acepción restringida, diversos estudios y análisis muestran su asociación con comportamientos de riesgo (rezago escolar, cesantía, madres adolescentes, drogadicción, delincuencia etc.) a los cuales se agregan otros efectos como la contracción de las redes sociales, el endurecimiento de las distancias sociales, y en general aislamiento de los pobres urbanos, esto es, la reproducción ampliada de la diferenciación social en donde el condicionamiento espacial es uno de los factores que incide en ella.

Diversos autores (CEPAL 2000d, Contreras1991), entre otros, constatan el aumento de la segregación socioespacial en A.Latina, tanto en su acepción amplia como la restringida, si bien el fenómeno asume características globales.

1 En palabras de Touraine refiriéndose a este problema; Un ejemplo, muy conocido por todos, es el de Los Ángeles -centro urbano muy limitado- con una serie de guetos en los que para pasar de un gueto a otro no hay otra solución que la autopista. Una autopista con guetos es, en mi opinión, una buena descripción del mundo actual. No hay comunicación, salvo la comunicación que todos conocemos: asaltos, guerra civil, racismo, xenofobia, etc. Cada grupo desprecia o tiene miedo de grupos nacionales, étnicos, religiosos, etc. Me parece el fin o la decadencia de la ciudadanía. En gran número de casos no somos más ciudadanos, sino más bien habitantes: gente que vive en un barrio, en un distrito, en una zona, en un edificio, etc. 
El aumento de la segregación socioespacial es, sin embargo, más acusado en la región. Por una parte, porque está asociado a la implementación de reformas neoliberales que han significado el aumento de la desigualdad social sobre la base de una desigualdad social preexistente, la liberalización del mercado de tierras y el abandono por los estados nacionales de los roles distributivos y proveedores de servicios a través de las políticas sociales, y por otra, por las consecuencias directas o indirectas de las políticas urbanas prevalecientes.

En América Latina la realización de reformas neoliberales es el aspecto más importante de su vida económica y social en las últimas décadas y una de sus consecuencias, como se señaló anteriormente, ha sido el aumento de la desigualdad social. Si tomamos la distribución desigual del ingreso como uno de los factores que incide en la segregación espacial, encontramos que en la región se encuentran una de las mayores disparidades en la distribución del ingreso en el mundo y que éstas están en aumento. ${ }^{2}$

En lo que se refiere a las políticas urbanas, las autoritarias e intencionales de erradicación de poblaciones asentadas al interior del perímetro urbano hacia la periferia se traducen directamente en segregación. Un ejemplo de esta intervención urbana fue la del gobierno militar en Chile, el cuál introdujo un proceso de relocalización espacial en un caso extremo de segregación socioespacial. Las políticas implementadas desde 1979 a través de la asignación diferencial de los recursos a los municipios de Santiago, y el desplazamiento de los habitantes más pobres a las zonas más alejadas de la ciudad con el consiguiente acceso diferencial a bienes y servicios públicos, confluyeron en una mayor desigualdad y segregación. (Morales, 1989) Estas políticas al parecer no son exclusivas de los gobiernos autoritarios.

En segundo lugar, las políticas de planificación institucional del zoning de producción en términos de la división funcional del espacio, por lo general, se han traducido indirectamente o directamente en segregación.

Un autor (Oszlack,1982) demuestra como políticas de planificación sobre el espacio urbano produjeron un desplazamiento espacial de los sectores populares residentes en el área metropolitana bonaerense. Medidas tales como liberación de los pre-

2 Un cuarto del ingreso nacional en A.L. pertenece al $5 \%$ de la población más rica y el $40 \%$ del total del ingreso va al $10 \%$ de la población más rica. En el sureste asiático el $5 \%$ de los más ricos recibe el $16 \%$ en, el mundo en desarrollo esta cifra es del 13\%. La contraparte de esta concentración de los ingresos en A.L; el $30 \%$ de la población más pobre recibe sólo el $7.5 \%$ del ingreso total, cifra menor que cualquiera parte del mundo, que está por encima del $10 \%$. Comúnmente el coeficiente de Gini usado como indicador para medir la desigualdad del in greso varía entre 0.25 y 0.60 . En A.L. Se encuentra aproximadamente en el $0.52 \%$, con un mínimo de $0.43 \%$ para Uruguay y un máximo de 0.59 para Brasil. El $10 \%$ de los más pobres ha sufrido un $15 \%$ de perdida de sus ingresos entre 1990 y 1995 y el siguiente $10 \%$ una pérdida de un $4 \%$. (Kloschkovsky, 2001). 
cios de los arriendos, códigos de edificación, erradicación de villas de emergencia, expropiación para construcciones de autopistas, recuperación de espacios verdes y relocalizaciones industriales centrifugan habitantes pobres de las zonas urbanas privilegiadas. Los efectos de estos procesos se traducen en encarecimientos de arriendos urbanos, reducción de la oferta de viviendas o aumento de su precio; traslado de las fuentes de trabajo para los obreros y expulsión de los habitantes más pobres a la periferia,

En otras ocasiones las políticas de planificación urbana tienen como finalidad la mantención de las segregaciones socioespaciales. El plan Brunner - Humeres para Santiago (1934) patrocinaba la distribución espacial de los estratos sociales.(Armando de Ramón,1985)

Igualmente, las políticas de vivienda social se traducen en segregación cuando se localizan en lugares periféricos donde los precios de los terrenos son bajos entre otras razones por la ausencia o baja calidad de los equipamientos urbanos.

Estas políticas se reproducen en un gran número de casos en las ciudades de América Latina.

En estas condiciones tanto el mercado que asume un rol dominante en la estructuración del espacio urbano en el orden neoliberal como las políticas de planificación urbana, de vivienda y erradicaciones forzadas de poblaciones se traducen en segregación residencial en el sentido de división social del espacio, tanto en el aumento de las segregaciones prevalecientes y espacialmente concentradas en áreas de la periferia urbana o en el centro-ciudad deteriorado, como en la emergencia de nuevas formas de segregación socioespacial, gentrificación y autosegregación de los grupos positivamente privilegiados.

Estos procesos económicos y políticos conducen a una segregación compulsiva, son procesos coactivos exteriores a los individuos y se imponen a ellos, como coacción directa en distintos grados de violencia para los grupos no privilegiados o como corrientes sociales que condicionan elecciones para los grupos privilegiados.

\section{Las formas de la segregación socioespacial}

En América Latina la segregación de los pobres y excluidos comprende dos formas típicas:

1. - Periferia suburbana pobre. Sus atributos son viviendas precarias o de bajo confort; insuficiencia de transportes; servicios urbanos deficitarios. Estos espacios urbanos son informales cuando se trata de ocupaciones ilegales de terrenos o residencias y desde el punto de vista de la urbanización cuando no cumplen las condiciones urbano ambientales para ser usadas como residenciales. Tanto las poblaciones - marcos legales formalizados pero en viviendas industriales de bajo confort - como los campamentos - hábitat 
informal y precario- son las modalidades recurrentes de la segregación periférica, ambas en situación de subequipamiento urbano. Todas las grandes ciudades de A. Latina tienen periferias con estos atributos, localizadas más bien en determinadas áreas geográficas y no tanto en anillos o círculos concéntricos. Menos recurrente es la localización de campamentos y poblaciones al interior del perímetro urbano.

2. -Asentamientos de poblaciones populares y marginales en áreas centrales en proceso de deterioro urbano; edificios antiguos; deterioros de estructura; falta de condiciones de confort.

La composición social de las áreas segregadas va a estar determinada por el estadio de la transición urbana en se encuentren las ciudades latinoamericanas, un componente urbano predominante en las transiciones urbanas avanzadas y un componente principalmente de origen rural en las rezagadas.

\section{Segregaciones emergentes}

3. -La gentrificación consiste en la recuperación de los centros históricos antiguos por las clases medias y altas acompañado de la evicción de los habitantes pobres del centro ciudad hacia la periferia. En otras ocasiones la gentrificación coexiste con poblaciones marginales y excluidas a veces de edad avanzada(segregación demográfica) concentrada en manzanas o agrupaciones de manzanas. No se trata aquí de barrios heterogéneos y pluriclasistas sino una composición de segmentos socioespaciales. Los factores que favorecen estos procesos son la degradación / devaluación de la ciudad central, la existencia de numerosas viviendas vacantes y el rol de la administración municipal o y/o estatal como animadora de estos procesos de recualificación social (Lungo, 2000).

4-La auto segregación de los grupos altos hace referencia al asentamiento de comunidades de habitación amuralladas o enrejadas en la periferia o en barrios populares que tienen condiciones favorables de accesibilidad. Estos procesos que no favorecen la integración social. En el caso del establecimiento de comunidades cerradas de grupos sociales medios y altos en barrios populares, lo que se acentúa es el endurecimiento de las relaciones sociales en espacios urbanos de proximidad. Con ello algunos espacios urbanos o ciertas áreas de la periferia se privatizan al mismo tiempo que monopolizan la exclusividad social y la calidad de vida.

El fenómeno de la segregación residencial no es, desde luego, un hecho nuevo en las ciudades latinoamericanas; existe desde la Colonia, sin embargo, en los últimos años, la polarización económica y espacial de la población ha llevado a que las ciudades se vean amenazadas de fragmentación y no sólo un aumento de la segregación de los grupos pobres y excluidos. 


\section{Segregación socioespacial y politicas públicas}

El examen de las políticas públicas en A. Latina en relación a la segregación socioespacial encuentra serias dificultades. En primer lugar porque la institucionalidad encargada de elaborar y ejecutar las políticas urbanas es muy variada(Ministerios, organismos regionales, municipalidades y consejos institucionales en distintos grados de jerarquización y ámbitos de competencia) y en segundo lugar, por la ausencia de cuerpos legislativos unificados con relación a las políticas urbanas que puedan constituirse en hitos de referencia, Ej. inexistencia de códigos de urbanismo u otras modalidades de unificación de la legislación relativa a las problemáticas urbanas.

Sin embargo, el hecho central es la ausencia de políticas institucionales que aborden frontalmente este problema en el contexto de las políticas urbanas. Políticas orientadas a la reversión de la segregación existen sólo como recomendaciones de instancias institucionales como por Ej. las del Consejo Nacional de Población de Méjico que proponen políticas para revertir estos procesos en la región metropolitana mejicana (Recuadro 1) o como las de académicos, científicos, y profesionales, orientadas a la elaboración de políticas antisegregacionistas (Recuadro2.)

Al parecer sólo existen algunas disposiciones de carácter sectorial y dispersas en políticas de ámbitos de competencia diferentes. Ello demuestra en el contexto de América Latina la débil legitimidad de la segregación socioespacial como problema para los actores institucionales de la ciudad.

\section{Recuadro 1}

México (zona metropolitana): Propuestas de políticas para revertir el proceso de segregación socioespacial

En el marco de los escenarios demográficos y urbanos de la Zona Metropolitana de la Ciudad de México, 1990-2010, en la sección 8.2 se proponen cuatro lineamientos de políticas con relación a la segregación socioespacial:

a) Mejoramientos de infraestructuras y servicios sociales en las colonias populares en formación y en proceso de consolidación ${ }^{3}$ con ei fin de corregir las desigualdades actuales y centrando la acción en los grupos de menores ingresos.

3 Las colonias populares son asentamientos producidos por las diversas modalidades del poblamiento popular. Las " en formación " generalmente no cuentan con obras de uroanización o esta es mínima. Las en "proceso de consolidación " no cuentan con urbanización completa y coexisten construcciones provisionales con terminadas. 
b) Consolidación de la vivienda en las colonias populares y los pueblos conurbados ${ }^{4}$. Las colonias "en formación " constituyen el espacio prioritario.

c) Conservación y creación de formas de poblamiento socialmente heterogéneas, particularmente en las colonias populares de la ciudad central. Las políticas de redensificación de las áreas de más antigua urbanización permitiría contener el traslado de la población, en particular, las colonias populares. Las herramientas de esta políticas pueden encontrarse en el programa de renovación habitacional popular aplicado después de los sismos de 1985. Este programa muestra que la conservación del uso habitacional en las áreas centrales dependió de una medida excepcional -la expropiación masiva de predios- y la disposición a subsidiar el costo del suelo para los beneficiarios del programa.

d) Reformulación de la acción habitacional pública sobre la base de seis propuestas:

1.- Adecuar las políticas de vivienda a las economías de los hogares, en particular a los de menores ingresos.

2.- Programar la vivienda y el hábitat popular.. El 60\% de la futura demanda de vivienda corresponderá a las necesidades habitacionales de familias de bajos ingresos.

3.- Subsidiar la producción de soluciones habitacionales alternativas a la urbanización irregular.

4.- Impulsar programas habitacionales mixtos.

5.- Vincular la producción y mejoramiento del hábitat popular con la generación de empleo, el combate a la pobreza y la integración comunitaria.

6.- Crear un instituto metropolitano de la vivienda para lograr un poblamiento equilibrado del Valle de México.

Fuente: CONSEJO NACIONAL DE POBLACIÓN. Escenarios demográficos y urbanos de la Ciudad de México, 1990-2010. Primera edición: septiembre 1998.

Estas recomendaciones que se inscriben en el marco de la zona metropolitana de Ciudad de Méjico, basan sus medidas antisegregacionistas en una redistribución de los equipamientos urbanos, en la mantención de la heterogeneidad social del

4 Los pueblos conurbados son aquellos que se desarrollaron en el pasado separados del espacio urbano pero conectados con este por las vias de comunicación y que en las cuatro últimas décadas pasaron a formar parte del área urbana de la Zona Metropolitana. 
centro urbano y en políticas habitacionales orientadas al hábitat popular, en lo posible programas mixtos -heterogeneidad social- $y$ a soluciones alternativas a la urbanización irregular.

En el recuadro 2 se exponen medidas relativas a revertir la segregación informal o irregular, generalmente hábitat suburbano periférico. Básicamente se trata de medidas de intervención en el mercado de tierras urbanas y proposiciones de leyes y marcos legales de regularización de la situación informal.

\section{Recuadro 2}

América latina: Propuestas de políticas para intervenir en el hábitat informal urbano.

Es informal este tipo de hábitat cuando desde el punto de vista

1.- Dominial hay:

a) Ocupación de tierra pública o privada y construcción ilegal

b) La tierra es legal pero la construcción es ilegal, es decir, sin las normas que los códigos de construcción establecen.

c) Ocupación ilegal de terrenos pero construcción legal. p. e. Compra de una casa prefabricada.

2. - De la urbanización la ocupación de tierras carece de condiciones ambientales para ser usadas como residenciales.

Las propuestas políticas significan intervenir en dos niveles: ayudar al acceso a la tierra en el mercado formal a la población pobre y regularizar las situaciones de informalidad existentes.

En relación al primer nivel se proponen:

1.1 Dinamización del mercado formal de tierras destinado a los sectores de más bajos ingresos con el fin de lograr densidades más altas y reducir los costos sociales y económicos asociados a la baja densidad de ocupación. Se proponen diversas medidas reguladoras en este sentido.

1.2 Constitución de un Banco de Tierras con tierras públicas y privadas.

1.3 Reformulación de instrumentos normativos, en particular el de zonificación por la relación que tiene con los procesos de segregación.

1.4 Política fiscal que, por una parte, aumente el impuesto inmobiliario a los 
lotes vacantes, y por otra, genere impuestos que permitan captar la valorización producida por cambios de la zonificación e inversiones públicas.

1.5 Fortalecimiento institucional para llevar a cabo las políticas anteriormente mencionadas.

1.6 Fortalecimiento de la población promoviendo su participación y asesorándola en término técnicos y jurídicos.

Con relación al segundo nivel se proponen elaboración de planes de regularización que, entre otros aspectos implica los siguientes

2.1 Disponibilidad de tierras e intervención en el mercado por; El uso del derecho preferencial de compra; legalización de asentamientos sobre tierras municipales y otros emergentes.

2.2 Fortalecer los organismos locales y agilizar trámites, por ejemplo de regularización dominial, desbaratamiento de mecanismos clientelísticos que no contribuyen a la transparencia de los proyectos.

2.3 Diseño de políticas financieras.

2.4 Definición de normativas urbanas y ambientales a aplicar a los programas y regularización diferenciada según los tipos de informalidad.

2.5 Mejorar la participación comunitaria en términos reales y

2.6 Minimizar los costos legales asociados a la legalización.

Fuente : Nora Clichevsky. Informalidad y segregación urbana en América Latina. División del Medio Ambiente y Asentamientos humanos. CEPAL. Santiago de Chile, octubre 2000.

El hecho de que no se encuentran políticas institucionalmente planificadas antisegregacionistas en América Latina no quiere decir que estas no existan en otras áreas regionales. Las experiencias socialdemócratas europeas y especialmente las nor europeas son un testimonio de ello. En este sentido recojo la ponencia presentada al seminario internacional de segregación en la ciudad (Lincon Institute, 2000) de René B, J. Teule, Primer consejero de la ciudad de La Haya. En esta ponencia el autor analiza la política holandesa orientada a objetivos antisegracionistas. Esta es una política de estado que articula la planificación física del territorio con la regulación urbana y la política de la vivienda gestionada por las corporaciones municipales. Teniendo como instrumento central la planificación institucional en los niveles señalados, un gran ámbito de competencia de las municipalidades y el control del mercado de la tierra, Holanda ha desconcentrado las ciudades y limitado el crecimiento de las ciudades más gran- 
des (Ámsterdam y Rótterdam no alcanzan a los 800.000 habitantes), ha eliminado las suburbanizaciones periféricas y ha favorecido el desarrollo de barrios socialmente heterogéneos, no obstante algunos resultados no queridos asociados a las inmigraciones más recientes.

\section{Recuadro 3}

\section{Holanda: Una propuesta de políticas antisegregacionista}

Las políticas antisegregacionistas incluyen:

a)Las políticas de la vivienda.- En Holanda el derecho a la vivienda es un derecho reconocido en la Constitución. Este derecho ha sido un factor significativo en la distribución igualitaria de la población dentro de las ciudades. Basada en la ley de vivienda de 1901 los elementos más importantes de este sistema han sido:

1. - La institucionalización de la gestión de las viviendas por las corporaciones habitacionales cuyo origen data del siglo xix. Por medio de esta ley las corporaciones se enmarcaron en la institucionalidad gubernamental, las políticas habitacionales entraron a ser parte del bien común y el gobierno retribuyó financieramente a las corporaciones que ocuparon un rol central en los planes habitacionales del sector público. En estas políticas los grupos vulnerables están protegidos por las políticas sociales impidiendo cualquier selección de riesgo entre los arrendatarios

2. - Subsidio orientado a compensar los bajos ingresos. El cálculo del subsidio se efectúa estableciendo un límite del valor de la vivienda y un límite de lo que se puede pagar. Las viviendas de menor valor tienen una calidad básica que es bastante buena en los países bajos. Hay también un sector privado de la vivienda para grupos de mayor ingreso aunque está bastante regulado.

3. -Distribución de la vivienda por parte de las municipalidades en el marco de las políticas estatales y municipales y no por la asignación del mercado. Esta implica control de las habitaciones y de los habitantes, establece precios máximos para arriendos y compras de propiedades. En general, las municipalidades controlan el mercado inmobiliario.

b) La planificación urbana y la planificación del territorio.- Consiste en la conexión de las políticas habitacionales con la regulación urbana y a su vez 
esta última articulada con la regulación nacional del espacio.. El concepto central que orienta estas regulaciones en el ámbito nacional es la desconcentración de la población en racimos sin que estos dependan de los servicios urbanos, esto es, con sus propios equipamientos básicos. Estos nuevos pueblos evitarían los riesgos de la suburbanización que no ha sido planificada

El Vinex es una situación de grupos de vivienda cerca de las ciudades que asocian habitación con proximidad del lugar de trabajo y equipamientos públicos. Son unidades urbanas más tardías y se localizan entre los nuevos pueblos y las grandes ciudades.

c) Renovación urbana.-Hace referencia a la demolición de las habitaciones viejas y pobres de preguerra reemplazándolas por nuevas construcciones, abriendo espacio para nuevas viviendas y contribuyendo de esta manera a evitar la suburbanización no planificada. Dada las políticas de la vivienda prevalecientes, la renovación no implican la evicción de los habitantes de menores ingresos.

Fuente : Dr René B.J. Teule MPA. Primer consejero. Departamento de Desarrollo Urbano. La Haya. Documento presentado al International Seminar on Segregation in the City.Julio 2000.Lincoln Institute.

Si bien en América Latina no se encuentran políticas institucionales orientadas a revertir la segregación, hay una experiencia notable de política municipal de democracia participativa que no teniendo como objetivo explícito la antisegregación, al desplazar decisiones y orientación de políticas urbanas de la institucionalidad municipal a la participación ciudadana se han traducido en limitaciones de los efectos de la segregación.

En Porto Alegre, Brasil, el gobierno municipal redefine la democracia local orientándola hacia la participación ciudadana. El instrumento principal de la democracia participativa es el "presupuesto participativo", sistema mediante el cual miles de residentes pueden participar cada año en asambleas públicas para decidir el destino de la mitad de los fondos presupuestarios municipales, asumiendo así una mayor responsabilidad por el gobierno de su propia comunidad. Los vecinos toman decisiones sobre asuntos locales prácticos y también sobre cuestiones más complejas que atañen a la ciudad. Diversos "temas prioritarios" se refieren a la segregación socioespacial. 


\section{Recuadro 4}

\section{Una evaluación señala que}

'En 1989, pese a sus altos índices comparativos de alfabetismo y esperanza de vida, las condiciones en Porto Alegre reflejaban la desigualdad y segregación económica de otras ciudades brasileñas. Un tercio de la población vivía en barrios bajos de la periferia urbana carentes de servicios básicos, aislados y distantes de la zona pudiente en el centro de la ciudad". "Entre 1988 y 1997 el suministro de agua a los hogares de Porto Alegre pasó de un 75 por ciento a un 98 por ciento de todas las residencias; el número de escuelas se ha cuadruplicado desde 1986; se han construido nuevas unidades de vivienda pública (éstas albergaban apenas 1700 nuevos residentes en 1986, frente a 27 000 residentes adicionales en 1989); a través de la intervención municipal se facilitó un arreglo con compañías autobuseras privadas para que mejoraran el servicio prestado a las vecindades periféricas de escasos recursos".

Fuente: William Goldsmith and Carlos Vainer. Presupuesto politico y poder en Puerto Alegre. Land lines 2001

En síntesis, la reconfiguración de los poderes locales está contribuyendo a reducir las desigualdades socioespaciales mediante cambios en los patrones de provisión y uso del suelo, al mismo tiempo que favorece el acceso al poder local de los grupos tradicionalmente excluidos y segregados.

La participación de los ciudadanos en el poder local ha aumentado significativamente. De 1000 en 1990 pasó a 16000 en 1998 y a 40000 en 1999.

\section{Bibliografía de referencia}

Achig S., Lucas A.(1986) Le processus urbain de Quito.

En: Delaunay, Daniel, comp. Portais, Michel,comp...Equateur 1986. Paris:ORSTOM.1989

Barco de Botero, Carolina y Ralph Gakenheimer (2000) Bogotá. La expansión del uso de la tierra ¿Puede ser controlada?.Lincoln Institute of Land Policy. Cambridge.U.S.A.

Borja, Jordi y Manuel Castells(2000. La ciudad multicultural. (www, aquibaix.com/ factoría/artículos/borjas $2 . \mathrm{atm})$ 
Clichevsky Nora, (2000) Informalidad y segregación urbana en América Latina. Una aproximación". CEPAL. Santiago de Chile

Deler, Jean Paul. Un espace marqué par la metropolisation. Problemes d Amerique Latine, $\left.\mathrm{n}\right|^{\circ} 14$

De Ramón, Armando. Santiago de Chile. Mapfre. Santiago de Chile 1985.

Fadda Cori, Giuliettta. (1996) La ciudad: una estructura multifacética. Valparaíso, Universidad de Valparaíso.

Goldsmith William and Carlos Vainer. (2001) Presupuesto político y poder en Puerto Alegre Land Lines 2001.Lincoln Institute, Cambridge.U.S.A.

Kaztman Rubén (2001) Seducidos y abandonados: pobres urbanos, aislamiento social y políticas públicas. CEPAL. Santiago de Chile

Klochkovsky Lev.L. Una vuelta por el mundo- Consecuencias sociales de las reformas neoliberales en América Latina y Rusia. Un análisis comparado de estadísticas básicas. Institute for Latin American Studies. Moscú, 2001.

Lefebvre Henri. El derecho a la ciudad. Ediciones Península. Barcelona 1969

Lincoln Institute of Land Policy, Ponencias presentadas al international seminar on segregation in the city, Cambridge, ma july 25-28, 2001.U:S:A: ___Correa do Lago, Luciana (Brazil) "Socio-spatial structuring in greater metropolitan Rio de Janeiro: a reproduction or transformation of conditions in the (lack of) access to urban space?"

Lungo ,Mario (El Salvador) Socio-spatial segregation and urban land regulation in latin american cities.

-Machado Barbosa, Eva (Brazil) Urban spatial segregation and social differentiation: foundation for a typological analysis"

Teule, René (Netherlands) Segregation in the low countries: a marketoriented dutch polder model as a solution!?"

. Oszlack,Los sectores populares y el derecho al espacio urbano. Buenos aires. , 1982.

Mejía, Marco Vinicio(1992) Para entender la ciudad de Guatemala. Instituto nacional de estadísticas. Guatemala

México. Consejo Nacional de Población. (1998) Escenarios demográficos y urbanos de la zona metropolitana de la Ciudad de México, 1990-2010: síntesis. CONAPO.

Morales Eduardo. Segregación socioespacial en la ciudad de Santiago. Seminario sobre Latina. Quito 1987. 
Rodriguez Vignoli, Jorge.(2001)Segregación residencial socioeconómica: ¿Qué es?,¿Cómo se mide?, ¿Qué está pasando?, ¿Importa?.Celade-Fnuap. Santiago de Chile

Sabatini, Francisco (1997): Liberalización de los mercados del suelo y la segregación de I en las ciudades latinoamericanas social: el caso de Santiago, Lincoln Institute de Política de la Tierra, Cambridge.

Touraine Alain. La transformación de las metrópolis. Este artículo es la transcripción de la conferencia que Alain Touraine pronunció el pasado dia 2 de febrero de 1998 en Barcelona con motivo del "10è aniversari de la Mancomunitat Metropolitana".

Van Lindert, Paul; Verkoren, Otto. (1982) Segregación residencial y política urbana en la Paz, Bolivia. Boletín de Estudios Latinoamericanos y del Caribe, $n^{\circ} 33$.

Vidal-Koppmann, Sonia. (2001) Segregación residencial y apropiación del espacio: la migración hacia las urbanizaciones cerradas del área metropolitana de Buenos Aires (Argentina)

En Revista Electrónica de Geografia y Ciencias Sociales Universidad de Barcelona [ISSN 1138-9788] No 94 